\title{
Optomechanically induced transparency and nonlinear responses based on graphene optomechanics system
}

\section{Hua-Jun Chen ${ }^{1 *}$ (10}

\section{*Correspondence:}

chenphysics@126.com

'School of Mechanics and

Photoelectric Physics, Anhui

University of Science and

Technology, Huainan Anhui, China

\begin{abstract}
Due to its unique properties of low mass density, high frequency, high quality-factor and intrinsically small size, graphene has received significant attention and is considered as an ideal material for fabricating nanomechanical systems. In this work, we demonstrate theoretically the coherent optical propagation under different detuning regimes in the graphene resonator-microwave cavity system. In the red detuning, optomechanically induced transparency induced the slow light effect is investigated and the maximum group delay can reach $0.4 \mathrm{~ms}$. In the blue detuning, advancing microwave signal can reach $0.12 \mathrm{~ms}$, and the parametric amplification process can behave as an optical transistor to amplify a weak microwave field via manipulating the pump field. Further, we research the nonlinear effects of four-wave mixing (FWM), and the FWM intensity can be efficiently controlled and modulated by the pump power, such as FWM spectrum can present the phenomenon of normal mode splitting with controlling the pump power. A straightforward optical means for determining the frequency of the resonator by the FWM spectrum is also presented. The graphene optomechanics may indicate applications in quantum information processing.
\end{abstract}

Keywords: Graphene optomechanics; Optomechanically induced transparency; Slow and fast light; Four-wave mixing

\section{Introduction}

Graphene, atomically thin two-dimensional (2D) nanomaterial consisting of a single layer of carbon atoms, has received tremendous attention in recent years. Due to its unique properties of low mass density, high frequency, high quality-factor and intrinsically small size, graphene may be considered as the ultimate nanomaterial for fabricating nanomechanical systems (NMSs) [1-4] to investigate the quantum behavior of the motion of micromechanical resonators. Due to these properties, graphene-based NMSs have potential applications in force detection [5-7] and mass sensing $[8,9]$. However, all these scientific applications are accomplished still in the classical regime at present. Although the detection and characterization of graphene resonators based on the previous optical method [5] and radiofrequency techniques $[1-3,10]$ have been demonstrated, how to provide a clear path towards the quantum regime is still a challenge. Reaching the quantum regime with mechanical resonators has attracted considerable interest [11] at present.

(c) The Author(s) 2019. This article is distributed under the terms of the Creative Commons Attribution 4.0 International License (http://creativecommons.org/licenses/by/4.0/), which permits unrestricted use, distribution, and reproduction in any medium, provided you give appropriate credit to the original author(s) and the source, provide a link to the Creative Commons license, and indicate if changes were made. 
Three groups using a piezoelectric resonator [12], a superconducting resonator [13], and an opto-mechanical crystal [14], respectively, have been successful demonstrated that the number of vibrational quanta of the nanoresonator can be lowered below one. Photothermal interaction between the motion of a mechanical resonator made with graphene and light was observed [15], where an on-chip trench covered by graphene to form a optical cavity. However, since the graphene end mirror is $98 \%$ transparent, the quality factor value $Q$ of the graphene resonator was much less than 1 . Thus the canonical radiation pressure phenomena observed in typical cavity optomechanical system (C-OMS) [11, 16-18] is hard be observed in graphene resonator system [15].

On the other hand, optomechanics systems (OMS) [11], investigating radiationpressure-induced coherent photon-phonon interactions, have drawn one's attention due to they pave the way for potential applications of optomechanical devices, such as phonon laser [19, 20], the realization of squeezed light [21-23], and ground-state cooling [12-14]. Another remarkable advance discovered in the OMS is optomechanically induced transparency (OMIT) [24-28], which provides a new way to store light in solid-state devices $[29,30]$. Additionally, mechanically-mediated delay (slow-light) and advancement (fastlight) of signals based on OMIT have been observed both in optical [27] and microwave domains [29, 31], which will offer new prospects for on-chip solid-state architectures capable of storing, filtering, or synchronizing optical light propagation.

Due to the radiation pressure in graphene resonator system is more weaker than in OMS [11], to amplify the radiation pressure, fabricating cavity optomechanics systems with graphene resonators and high quality factors superconducting microwave cavity are a natural candidate. Multilayer graphene resonators with high-Q capacitively coupled to superconducting microwave cavities have been demonstrate experimentally currently [32, 33]. Compared with monolayer graphene, multilayer graphene owns the virtue of lower electrical resistance, and the atomically thin 2D shape of graphene is ideal for large capacitive coupling, which is advantageous for coupling to a superconducting cavity, although multilayer graphene has higher mass than a monolayer. Additionally, graphene optomechanics, in which the interaction between light and micromechanical motion via the radiation pressure, based on a bilayer graphene resonator coupled to a microwave on-chip cavity has been investigated experimentally most recently [34]. Therefore, the realization of graphene optomechanics in experiment is promising for reaching the quantum regime of graphene motion. If the graphene optomechanical cavity system [34] is driven by a strong pump field with frequency $\omega_{p}$ and a weak probe field with frequency $\omega_{s}$, the coherent propagation properties such as optomechanically induced absorption (OMIA) and optomechanically induced reflection (OMIR) can appear in the system due to the optical radiation pressure [33]. Besides the fields at the driven field frequencies $\omega_{p}$ and $\omega_{s}$, a optical four-wave-mixing (FWM) field with frequency $2 \omega_{p}-\omega_{s}$ will also emerge in the graphene optomechanical system.

FWM, as typical nonlinear optical phenomenon, is investigated in OMS, such as the mode-splitting in strong coupling optomechanical system [35], coherent mechanical driving OMS [36, 37], and a two-mode cavity optomechanical system [38]. Usually, the power of the output FWM field is still much less than the power of the input probe field, as we know there is no experimental reports on the demonstration of FWM field in optomechanical systems up to now, and FWM slao have been seldom studied in graphene optomechanical system. 
In the present paper, we demonstrate the tunable ultraslow light effect based on the phenomena of OMIT [24-27] in the graphene nanomechanical resonator-microwave cavity system, and the maximum group delay of the transmitted probe field is about $0.4 \mathrm{~ms}$. The superluminal light effect induced by the photon-phonon interaction can also appear at the blue sideband, and the system can behave as a photonic transistor effectively controlling the propagation of the microwave field. Further, we theoretically investigated the FWM field in the graphene optomechanical system. The generated FWM field is induced by the radiation pressure force on the graphene mechanical resonator, the strength of the FWM field can be modulated by the pump field power, and a high FWM conversion efficiency can obtain at low pump power. Moreover, a nonlinear optical scheme for determining the frequency of a graphene resonator is also presented.

\section{System and method}

Graphene cavity optomechanical system [32-34], composed of a graphene mechanical resonator capacitively coupled to a high- $Q$ microwave cavity resonator denoted by equivalent inductance $L$ and equivalent capacitance $C$, is sketched in Fig. 1. In Fig. 1, the coupling capacitance can be approximated by $C_{g}(x) \sim \varepsilon_{0} h W /(d-x)$ where $h$ is the width of the suspended part of graphene, $W$ is the width of the gate below the graphene, $d$ is the narrow vacuum gap, and $x$ denotes the displacement of the maximum deflection point of the garphene membrane from the equilibrium position [34]. Therefore, the total capacitance $C_{\text {tot }}(x)$ of the coupled cavity can be summed up as a constant $C$ and a small $x$-dependent part $C_{g}(x)$. The resonance frequency of this microwave cavity hence is $\omega_{c}=1 / \sqrt{L\left(C+C_{g}(x)\right)}$. A strong pump field with frequency $\omega_{p}$ and a weak probe field with frequency $\omega_{s}$ are applied to the microwave cavity simultaneously. The beating of the two fields induces the graphene mechanical resonator to vibrate, which can change the capacitance of the microwave cavity and thus its resonance frequency. In a rotating frame of the pump frequency $\omega_{p}$, the Hamiltonian of the system as follows [31, 34, 39]

$$
\begin{aligned}
H= & \hbar \Delta_{p} a^{+} a+\hbar \omega_{m} b^{+} b-\hbar g a^{+} a\left(b^{+}+b\right) \\
& +i \hbar \sqrt{\kappa_{e}}\left(E_{p} a^{+}-E_{p}^{*} a+E_{s} a^{+} e^{-i \Omega t}-E_{s}^{*} a e^{i \Omega t}\right) .
\end{aligned}
$$

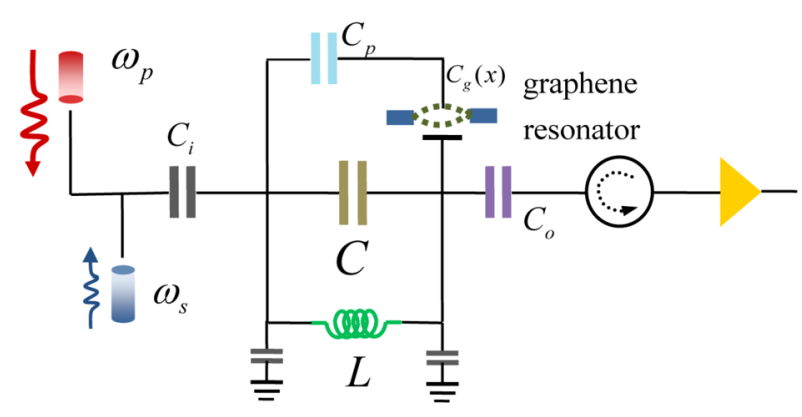

Figure 1 Schematic of a graphene mechanical resonator capacitively coupled to a microwave cavity denoted by equivalent inductance $L$ and equivalent capacitance $C$ in the presence of a strong pump field $\omega_{p}$ and a weak probe field $\omega_{s}$. The transmitted probe field can be detected at room temperature by a spectrum analyzer 
The first term is the energy of the microwave cavity with the creation (annihilation) operator $a^{+}(a)$ of the microwave cavity and $\Delta_{p}=\omega_{c}-\omega_{p}$ is the cavity-pump field detuning. The second term indicates the energy of the graphene mechanical resonator, resonance frequency $\omega_{m}$ and effective mass $m_{\text {eff }}$. The third term corresponds to the capacitive coupling between the microwave cavity and the graphene mechanical resonator, where $g=\left(\partial \omega_{c} / \partial x\right) x_{z p}$ is the coupling strength between the cavity and the graphene resonator, and $x_{z p}=\sqrt{\hbar / 2 m_{\text {eff }} \omega_{m}}$ is the zero-point motion of the nanomechanical resonator. Due to the properties of graphene, the value of $x_{z p}$ is nearly two orders of magnitude larger than the same number of metallic membrane resonators [13], which is beneficial for studying the quantum behavior of the motion of graphene mechanical resonators. The last two terms represent the interaction between the cavity field and the two driven optical fields, and $\Omega=\omega_{s}-\omega_{p}$ is the probe-pump detuning. $E_{p}$ and $E_{s}$ are the amplitudes of the pump field and the probe field, respectively, and they are defined by $\left|E_{p}\right|=\sqrt{P_{\text {in }} / \hbar \omega_{p}}$ and $\left|E_{s}\right|=\sqrt{P_{s} / \hbar \omega_{s}}$ where $P_{\text {in }}$ is the pump power, $P_{s}$ is the power of the probe field, and $\kappa_{e}$ is external loss rate.

Writing the Heisenberg equations of motion and adding dissipation of the corresponding damping and noise terms, the quantum Langevin equations of the system as follows,

$$
\begin{aligned}
& \dot{a}(t)=-\left(i \Delta_{p}+\kappa\right) a(t)+i g a(t) X(t)+\sqrt{\kappa_{e}}\left(E_{p}+E_{s} e^{-i \Omega t}\right)+\hat{a}_{\mathrm{in}}(t), \\
& \ddot{X}(t)+\gamma_{m} \dot{X}(t)+\omega_{m}^{2} X(t)=2 g \omega_{m} a^{+}(t) a(t)+\hat{\xi}(t),
\end{aligned}
$$

where the position operator $X=b^{+}+b, \gamma_{m}$ is the decay rate of the graphene resonator, and $\kappa$ is the decay rate of microwave cavity mode. $\hat{a}_{\text {in }}(t)$ is the $\delta$-correlated Langevin noise operator, which has zero mean $\left\langle\hat{a}_{\text {in }}(t)\right\rangle=0$ and obeys the correlation function $\left\langle\hat{a}_{\text {in }}(t) \hat{a}_{\text {in }}^{+}\left(t^{\prime}\right)\right\rangle \sim \delta\left(t-t^{\prime}\right)$. The resonator mode is affected by a Brownian stochastic force with zero mean value, and $\hat{\xi}(t)$ has the correlation function

$$
\left\langle\hat{\xi}^{+}(t) \hat{\xi}\left(t^{\prime}\right)\right\rangle=\frac{\gamma_{m}}{\omega_{m}} \int \frac{d \omega}{2 \pi} \omega e^{-i \omega\left(t-t^{\prime}\right)}\left[1+\operatorname{coth}\left(\frac{\hbar \omega}{2 \kappa_{B} T}\right)\right]
$$

where $k_{B}$ and $T$ are Boltzmann constant and temperature of the reservoir of the coupled system, respectively.

Since the probe field is much weaker than the pump field, following the standard methods of quantum optics, we can rewrite each Heisenberg operator as the sum of its steadystate mean value and a small fluctuation withs zero mean value: $O \rightarrow O_{s}+\delta O(O=a$, $X)$. Supposing the driving fields are weak but classical coherent fields, all operators can be treated with their expectation values, and the quantum and thermal noise terms will also be dropped [25]. In this case, the steady-state values are governed by the pump power and the small fluctuations by the probe power. Inserting these operators into the Langevin equations Eqs. (2)-(3) and neglecting the nonlinear term, we can obtain two equation sets about steady-state mean value and a small fluctuation. The steady-state equation set consisting of $a_{s}=\sqrt{\kappa_{e}} E_{p} /\left[i\left(\Delta_{p}-g X_{s}\right)+\kappa\right]$ and $X_{s}=2 g\left|a_{s}\right|^{2} / \omega_{m}$ are related to the intracavity photon number $\left(n_{0}=\left|a_{s}\right|^{2}\right)$ which is determined by $n_{0}\left[\kappa^{2}+\left(\Delta_{p}-2 g^{2} n_{0} / \omega_{m}\right)^{2}\right]=\kappa_{e}\left|E_{p}\right|^{2}$. For the equation set of small fluctuation, we make the ansatz [40]: $\langle\delta O\rangle=O_{+} e^{-i \delta t}+O_{-} e^{i \delta t}$. Solving the equation set and working to the lowest order in $E_{s}$ but to all orders in $E_{p}$, we 
can obtain

$$
\begin{aligned}
& a_{+}=\frac{\sqrt{\kappa_{e}} E_{s}}{\kappa+i(\bar{\Delta}-\Omega)-\frac{g^{2} \chi^{2} n_{0}^{2}}{\kappa-i(\bar{\Delta}+\Omega)}}, \\
& a_{-}=\frac{i g \chi^{*} a_{s}^{2} \sqrt{\kappa_{e}} E_{s}}{\left[\kappa+i\left(\bar{\Delta}^{\prime}+\Omega\right)\right]\left[\kappa-i\left(\bar{\Delta}^{\prime}-\Omega\right)\right]-g^{2}\left(\chi^{*}\right)^{2} n_{0}^{2}},
\end{aligned}
$$

where $\chi=2 g \omega_{m} /\left(\omega_{m}^{2}-\Omega^{2}-i \Omega \gamma_{m}\right), \chi^{*}=2 g \omega_{m} /\left(\omega_{m}^{2}-\Omega^{2}+i \Omega \gamma_{m}\right), \bar{\Delta}=\Delta_{p}-g X_{s}-g \chi n_{0}$, and $\bar{\Delta}^{\prime}=\Delta_{p}-g X_{s}-g \chi^{*} n_{0}$.

Using the standard input-output relation [41] $a_{\text {out }}(t)=a_{\text {in }}(t)-\sqrt{\kappa_{e}} a(t)$, we obtain

$$
\begin{aligned}
\left\langle a_{\mathrm{out}}(t)\right\rangle & =\left(E_{p}-\sqrt{\kappa_{e}} a_{0}\right) e^{-i \omega_{p} t}+\left(E_{s}-\sqrt{\kappa_{e}} a_{+}\right) e^{-i\left(\Omega+\omega_{p}\right) t}-\sqrt{\kappa_{e}} a_{-} e^{-i\left(\Omega-\omega_{p}\right) t} \\
& =\left(E_{p}-\sqrt{\kappa_{e}} a_{0}\right) e^{-i \omega_{p} t}+\left(E_{s}-\sqrt{\kappa_{e}} a_{+}\right) e^{-i \omega_{s} t}-\sqrt{\kappa_{e}} a_{-} e^{-i\left(2 \omega_{p}-\omega_{s}\right) t}
\end{aligned}
$$

where $a_{\text {out }}(t)$ is the output field operator. Equation (7) shows that the output field consists of three terms. The first term corresponds to the output field at driving field with amplitude $\varepsilon_{p}$ and frequency $\omega_{p}$. The second term corresponds to the probe field with frequency $\omega_{s}$ related to the anti-Stokes field resulting in OMIT, which has been investigated in various optomechanical systems [24-27]. The transmission spectrum of the probe field defined by the ratio of the output and input field amplitudes at the probe frequency, which shows

$$
t\left(\omega_{s}\right)=\frac{E_{s}-\sqrt{\kappa_{e}} a_{+}}{E_{s}}=1-\frac{\kappa_{e}}{\kappa+i(\bar{\Delta}-\Omega)-\frac{g^{2} \chi^{2} n_{0}^{2}}{\kappa-i(\bar{\Delta}+\Omega)}} .
$$

The transmission group delay can be expressed as [25]

$$
\tau_{g}=\left.\frac{d \phi}{d \omega_{s}}\right|_{\omega_{s=\omega_{c}}}=\left.\frac{d\left\{\arg \left[t\left(\omega_{s}\right)\right]\right\}}{d \omega_{s}}\right|_{\omega_{s=\omega_{c}}}
$$

The magnitude and phase of the transmitted probe could be determined experimentally by measuring the inphase and quadrature response of the system to the input modulation. The last one in Eq. (7) corresponds to the output field with frequency $2 \omega_{p}-\omega_{s}$ related to the stoke field displaying the FWM. In the FWM process the two photons of the driving field interact with a single photon of the probe field each with frequencies $\omega_{p}$ and $\omega_{s}$ born a new photon of frequency $2 \omega_{p}-\omega_{s}$. The FWM intensity in terms of the probe field can be defined as [38]

$$
F W M=\left|\frac{\sqrt{\kappa_{e}} a_{-}}{E_{s}}\right|^{2}=\left|\frac{i g \chi^{*} a_{s}^{2} \kappa_{e}}{\left[\kappa+i\left(\bar{\Delta}^{\prime}+\Omega\right)\right]\left[\kappa-i\left(\bar{\Delta}^{\prime}-\Omega\right)\right]-g^{2}\left(\chi^{*}\right)^{2} n_{0}^{2}}\right|^{2},
$$

which is proportional to the intracavity photon number $a_{s}^{2}$.

\section{Results and discussions}

We choose a realistic coupled nanomechanical resonator-microwave cavity system with the parameters as follows [34]: $m=10 \mathrm{fg}, m_{\mathrm{eff}}=0.4 \mathrm{~m}, C=45 \mathrm{fF}, C_{g}=0.4 \mathrm{fF}, \omega_{c} / 2 \pi=$ 

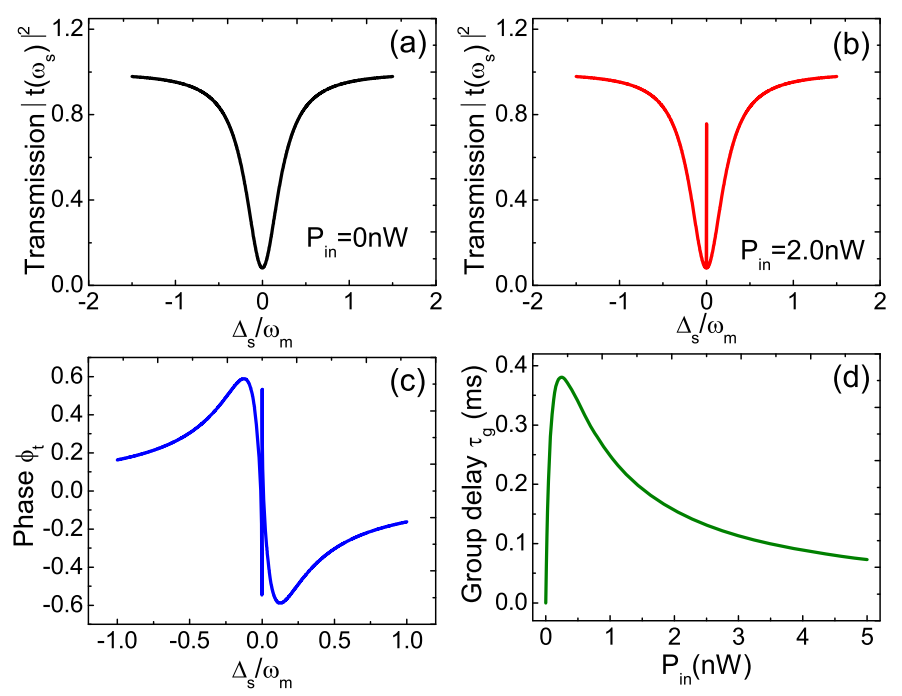

Figure 2 The normalized magnitude of the cavity transmission $|t|^{2}$ as a function of probe-cavity detuning $\Delta_{s}=\omega_{s}-\omega_{c}$ with the pump powers (a) $P_{\text {in }}=0$ (b) $P_{\text {in }}=3.0 \mathrm{nW}$, respectively. (c) The phase of the cavity transmission as a function of $\Delta_{s}$ for pump power $P_{\text {in }}=5 \mathrm{nW}$, and (d) the group delay as a function of the pump power $P_{\text {in }}$. All the plots are in the condition of red sideband $\Delta_{p}=\omega_{m}$

$7.82 \mathrm{GHz}, \omega_{m} / 2 \pi=24 \mathrm{MHz}, \kappa / 2 \pi=5.6 \mathrm{MHz}, g / 2 \pi=35 \mathrm{~Hz}$, the quality factor of the graphene mechanical resonator $Q=15 \times 10^{3}$, and $\gamma_{m} / 2 \pi=\omega_{m} / Q=1.6 \mathrm{kHz}$ in a dilution refrigerator down to the $22 \mathrm{mK}$ base temperature. We can see that $\omega_{m}>\kappa$ and therefore the system operates in the resolved-sideband regime.

We first consider the situation where the microwave cavity is driven on its red sideband, i.e., $\Delta_{p}=\omega_{m}$. Figure 2(a) and (b) plot the transmission $|t|^{2}$ of the probe beam as a function of the probe-cavity detuning $\Delta_{s}=\omega_{s}-\omega_{c}$ without $\left(P_{\mathrm{in}}=0 \mathrm{nW}\right)$ and with $\left(P_{\mathrm{in}}=2 \mathrm{nW}\right)$ the pump field powers. It is clearly see that the transmission spectrum of the probe beam shows a significant transparency window at $\Delta_{s}=0$ in the presence of a pump field, and the transparency window can be modulated effectively by the pump field, which has been demonstrated by several groups in optomechanical and electromechanical system [13, 25, 27]. This physical phenomenon is very similar to electromagnetically induced transparent. The simultaneous presence of pump and probe microwave fields induce a radiationpressure force oscillating at the beat frequency $\Omega=\omega_{s}-\omega_{p}$, which drives the graphene mechanical resonator near its resonance frequency. If the beat frequency $\Omega$ is close to the resonance frequency $\omega_{m}$ of the graphene resonator, the mechanical mode starts to oscillate coherently, which will result in Stokes $\left(\omega_{S}=\omega_{p}-\omega_{m}\right)$ and anti-Stokes $\left(\omega_{\mathrm{AS}}=\omega_{p}+\omega_{m}\right)$ scattering of light from the strong intracavity field. Since the cavity is driven on its red sideband, the Stokes scattering at the frequency $\omega_{p}-\omega_{m}$ is strongly suppressed and only the anti-Stokes scattering at the frequency $\omega_{p}+\omega_{m}$ builds up within the cavity. Destructive interference between the anti-Stokes field and the probe field can suppress the build-up of an intracavity probe field and result in the narrow transparency window, which indicates a tendency to reach the slow-light effect in graphene optomechanics. When the radiation pressure force drives the vibration of the graphene resonator, the displacement $x$ of the nanomechanical resonator from its equilibrium position will alter the capacitance of the microwave cavity and therefore its resonance frequency. The effective refractive in- 

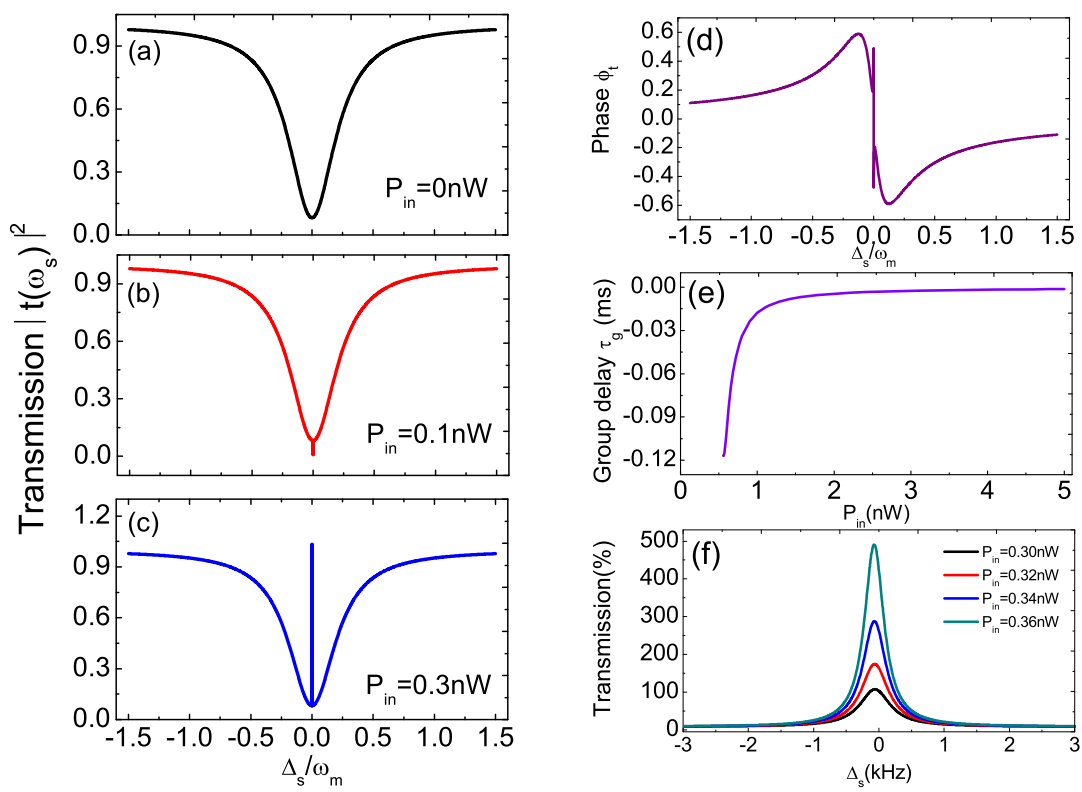

Figure 3 (a) to (c) show the normalized magnitude of the cavity transmission $|t|^{2}$ as a function of probe-cavity detuning $\Delta_{s}$ for three different pump powers $P_{\text {in }}=0,0.1,0.3 \mathrm{nW}$. (d) The phase of the cavity transmission as a function of $\Delta_{s}$ with pump power $P_{\text {in }}=5 \mathrm{nW}$, and (e) the group delay as a function of the pump power $P_{\text {in }}$. (f) The magnified transparency window as a function of $\Delta_{s}$ at $\Delta_{p}=-\omega_{m}$ for $P_{\text {in }}=0.3,0.32$, $0.34,0.36 \mathrm{nW}$, respectively. All the figures are at the condition blue sideband $\Delta_{p}=-\omega_{m}$

dex seen by the propagating probe field changes and a phase shift is induced under the red sideband. When the narrow transparency window appear in the graphene optomechanics, there will be a very steep positive phase dispersion around $\Delta_{s}=0$ at red sideband $\Delta_{p}=\omega_{m}$ with the pump power $P_{\text {in }}=5 \mathrm{nW}$, which will result in a tunable group delay (i.e. the slow-light effect) of the transmitted probe beam as shown in Fig. 2(c). In Fig. 2(d) we plot the transmission group delay $\tau_{g}$ of the probe beam versus the pump power $P_{\text {in }}$ under the red sideband $\Delta_{p}=\omega_{m}$. As can be seen from the figure, the maximum transmission delay is $\tau_{g} \approx 0.4 \mathrm{~ms}$, which is much bigger than in nanoscale optomechanical crystal system [27].

Switching the cavity-pump field detuning $\Delta_{p}$ from the red sideband $\Delta_{p}=\omega_{m}$ to the blue sideband $\Delta_{p}=-\omega_{m}$ and increasing the pump field intensity, we display the transmission spectra of the probe field as a function of $\Delta_{s}$ for three different pump field powers $\left(P_{\text {in }}=0\right.$, 0.1, $0.3 \mathrm{nW}$ ) as shown in Fig. 3(a)-(c). Under the blue sideband, the effective interaction Hamiltonian of the microwave cavity field and the graphene mechanical resonator mode reduces to $H_{\text {int }}=\hbar G\left(a^{+} b^{+}+a b\right)$ with the effective coupling strength $G=g \sqrt{n_{0}}$, which induces the parametric amplifications. When the control field is off $\left(P_{\mathrm{in}}=0\right)$, the transmission spectrum of the probe field shows the usual Lorentzian line shape of the cavity. As turning on the pump field, the transmission is attenuated around the probe cavity detuning $\Delta_{s}=0$ compared with the situation where the pump field is off at the pump power $P_{\text {in }}=0.1 \mathrm{nW}$. With further increase the pump field power to $P_{\text {in }}=0.3 \mathrm{nW}$, the system switches from electromagnetically induced absorption (EIA) to parametric amplification (PA) [27], inducing a signal amplification. Thus, the graphene optomechanical system can act as a photonic transistor, where the probe ('source') field is manipulated by the pump 
('gate') field. These phenomena can be understood as follows. The radiation pressure force drives the vibration of the graphene resonator near its resonant frequency under resonant cavity pump detuning, i.e., $\Delta_{p}= \pm \omega_{m}$. When the beat frequency $\Omega$ between the probe and pump field is close to the resonance frequency $\omega_{m}$ of the resonator, the mechanical mode starts to oscillate coherently, which will induce Stokes and anti-Stokes scattering of light from the strong intracavity field. When the microwave cavity is driven on its blue sideband $\left(\Delta_{p}=-\omega_{m}\right)$, it is the Stokes field that interferes with the near-resonant signal field and thus modifies the probe spectrum. Therefore, the constructive interference between the Stokes field and the probe field amplifies the weak probe field. Similarly, switching the cavity-pump field detuning $\Delta_{p}$ to the blue sideband $\Delta_{p}=-\omega_{m}$, we plot the phase of the transmitted probe field as a function of the probe-cavity detuning $\Delta_{s}$ at the pump power $P_{\text {in }}=5 \mathrm{nW}$ as shown in Fig. 3(d). Therefore a steep negative phase dispersion will appear at $\Delta_{s}=0$ which results in fast-light effect. Figure 3(e) presents the group delay $\tau_{g}$ as a function of the pump power for $\Delta_{p}=-\omega_{m}$. The group delay is negative, and then we can obtain the fast-light effect when the microwave cavity is driven on its blue sideband. Compared with the situation at the red sideband, we can propose a scheme to efficiently switch from pulse delay to pulse advancement by adjusting the pump-cavity detuning. Fixing the probe field with frequency at $\omega_{s}=\omega_{c}$ and then scanning the pump frequency across the microwave cavity resonance frequency $\omega_{c}$, one can efficiently switch from the probe pulse delay to advancement without appreciable absorption or amplification as the pump detuning $\Delta_{p}$ equals to $\omega_{m}$ or $-\omega_{m}$. We further focus on the narrow region around probe-cavity detuning $\Delta_{s} \approx 0$ which amplifies the probe field, and plot the transmission of the probe field as a function of the probe-cavity detuning in Fig. 3(f) for several pump field powers, respectively. It is clearly seen that the transmission is significantly enhanced around $\Delta_{s} \approx 0$ at the higher pump field power. Recently, similar results have been obtained experimentally [31]. Therefore, the transmitted probe field can be attenuated or amplified in this coupled system under the strong pump field when the cavity-pump detuning at the blue sideband $\Delta_{p}=-\omega_{m}$.

Actually, the similar results of Fig. 2 and Fig. 3 have been demonstrated in typical cavity optpmechanics [11] and graphene optomechanical system [33], here, we still give these results in this work because several reasons. The first one is that we are more care of the steep phase dispersion $\phi=\arg \left[t\left(\omega_{s}\right)\right.$ and its related coherent optical propagation under different detuning regimes, such as the slow light effect in red detuning and the fast light effect in blue detuning. When we investigate the coherent optical propagation (slow and fast light), the physical origin of the phenomenon of OMIT should be elaborated, and then we present OMIT as figure in Fig. 2(a) and (b). The second one is that we hope to present the whole information of the transmission spectrum at both the red and blue detuning. It is obvious that OMIT in red detuning induced the slow light, while OMIA and parametric amplification in blue detuning leading to the fast light, and a photonic transistor to amplify a weak microwave signal can also realize in blue detuning. Moreover, it is beneficial to make a contrast between Fig. 2 and Fig. 3. Compared with Fig. 2 and Fig. 3, we can clearly obtain the evolutionary process of the probe transmission spectrum, the steep phase dispersion, and the group delay $\tau_{g}$ at the two different detuning regimes. The third one is that the investigation of OMIT and OMIA is helpful for studying the nonlinear process of FWM. In the following, the nonlinear phenomenon of FWM process in the graphene optomechanics will be demonstrated emphatically, and the physical origin of FWM is at- 
tributed to the quantum interference, which also result in OMIT and OMIA. Due to the above reasons, we still present the results of Fig. 2 and Fig. 3 in this work although they are a little similar to the previous work [33].

On the other hand, as typical nonlinear optical phenomenon, we investigate the FWM process with Eq. (10) in the graphene optomechanical system. Figure 4 plots the FWM spectrum as a function of the probe-cavity detuning $\Delta_{s}=\omega_{s}-\omega_{c}$ at $\Delta_{p}=0$ for three different frequencies $\omega_{m}$ of the resonator, and the insert shows the amplification of the right three peaks. It is shown that three sharp peaks in the FWM spectra accurately locate at the resonance frequency of the resonator $\left(\Delta_{s}= \pm \omega_{m}\right)$. The physical origin of this result is attributed to the quantum interference between the mechanical modes and the beat of two optical fields via the cavity. When the beat frequency $\Omega$ of the two optical fields via the graphene optomechanical system is close to the resonance frequency $\omega_{m}$, the resonator starts to oscillate coherently, which results in Stokes $\left(\omega_{S}=\omega_{p}-\omega_{m}\right)$ scattering of light from the optomechanical system. Therefore, we can propose a straightforward optical scheme for determining the frequency of the resonator, and the measurement process includes two steps: (1) we apply a strong pump field to the system and fix the pump field at the resonance of cavity frequency $\Delta_{p}=0$; (2) we next apply another weak probe field to pass through the optomechanical system and detect the nonlinear transmission spectrum. Then two shark peaks will appear at the $\Delta_{s}= \pm \omega_{m}$ as shown in Fig. 4.

Switching $\Delta_{p}$ from $\Delta_{p}=0$ to $\Delta_{p}=\omega_{m}$, we further investigate the evolution process of the FWM spectrum as a function of the probe-cavity detuning $\Delta_{s}$ on the red sideband $\Delta_{p}=\omega_{m}$ as shown in Fig. 4. With increasing the pump power $P_{\text {in }}$, the FWM intensity near the resonant region is strengthened significantly, and the linewidth of the spectrum is broadened simultaneously as shown in Fig. 5(a). This is due to that more intracavity photon numbers are stimulated when the pump power increases, and thus the radiation pressure force and the effective coupling strength between the cavity and the resonator get stronger. On the other hand, without the interaction between the cavity field and the resonator, the FWM component will disappear immediately in the output field. Thus, the parametric coupling in the system plays a vital role in generating FWM, and the FWM

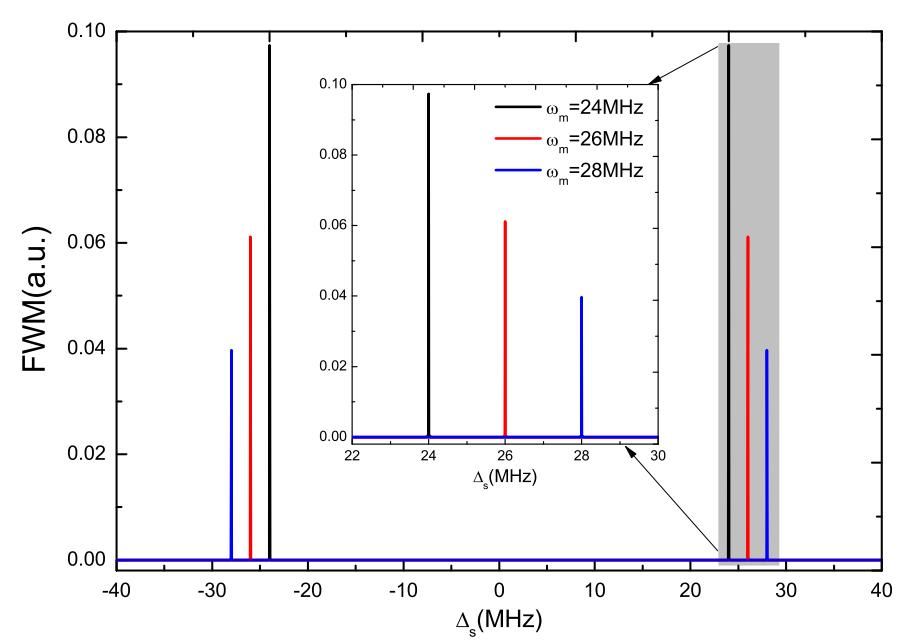

Figure 4 FWM intensity as a function of probe-cavity detuning $\Delta_{s}$ under resonance $\Delta_{p}=0$ for three different resonator frequencies 

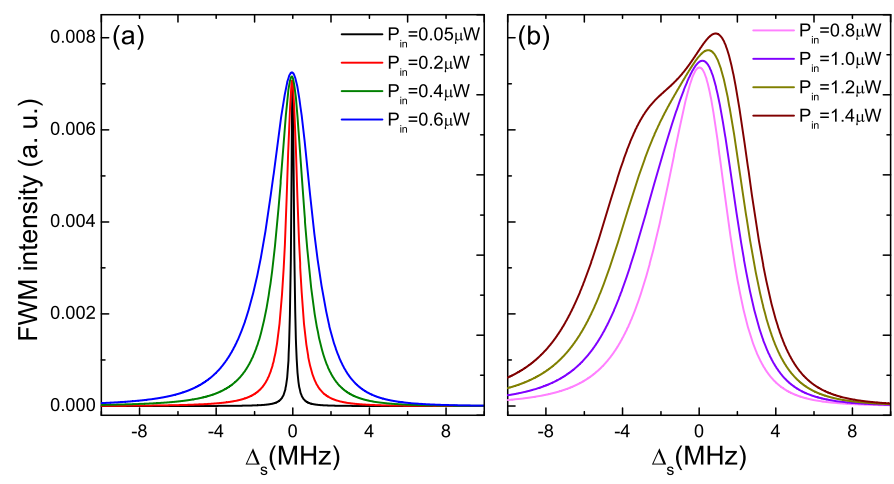

Figure 5 FWM intensity as a function of probe-cavity detuning $\Delta_{s}$ at $\Delta_{p}=\omega_{m}$ with several different pump powers

intensity increases when the cavity is driven by a stronger pump field. The physical origin is derived from the near-resonant probe field interfere with the anti-Stokes field $\left(\omega_{\mathrm{AS}}=\right.$ $\left.\omega_{p}+\omega_{m}\right)$, and thus the pump field and the probe field can mix well via the resonator. The magnitude of the mixture will enhance with increasing the pump power. However, when further increasing the pump power $P_{\text {in }}$ and reach a critical value, the process of FWM will change significantly as shown Fig. 5(b). We can see that the FWM spectrum will evolve from one peak to double peaks which is analogous the normal mode splitting, while the maximum value in the resonant region remains almost constant.

Return to Fig. 5(a), the peak value of FWM in the resonant region $\left(\Delta_{s}=0\right)$ is plotted as a function of the pump power under $\Delta_{p}=\omega_{m}$ as shown in Fig. 6(a), the FWM intensity near the resonant region is strengthened significantly and the linewidth of the spectrum is broadened simultaneously. With the increase of $P_{\mathrm{in}}$, this value is greatly enhanced and finally reaches saturation at a low pump power as shown in Fig. 6(b). Compared with the transmission spectrum $|t|^{2}$, the amplitude of the FWM spectrum is weak. In order to make the FWM phenomenon visible, two schemes can be introduced, The first one is that we can increase the pump power $P_{\text {in }}$. The Eq. (10) indicates the relation of $F W M \propto a_{s}^{2}$, and the intracavity photon number $a_{s}^{2} \propto P_{\text {in }}$. Then, if the pump power is enhanced, the intensity of the FWM can also improved. The second one is that we can pave one layer of metal nanoparticles (such as gold or silver) on the graphene. The metal nanostructures can be excited to produce surface plasmon (SP) with strongly enhanced electromagnetic fields, and surface plasmon provides the enhanced nonlinear optical effects.

The phenomena of OMIT and OMIA are ascribed to the quantum interference induced by the radiation pressure force, and the slow and fast light effects are demonstrated in the graphene optomechanical system, which may indicate applications in quantum information processing. On the other hand, this enhanced FWM may prove useful in generating nonclassical states, such as squeezed states of microwave fields, and generation and detection of the squeezed sates of the output microwave field has been proposed by parametrically driving the nanomechanical resonator capacitively coupled to a microwave cavity [42]. In addition, this extremely low power with a resonantly enhanced nonlinear effect of the graphene optomechanics may indicates potential applications in real communication networks. One potential application is microwave communication networks for frequency conversion at a low pump power. When the graphene resonator-microwave cavity system 

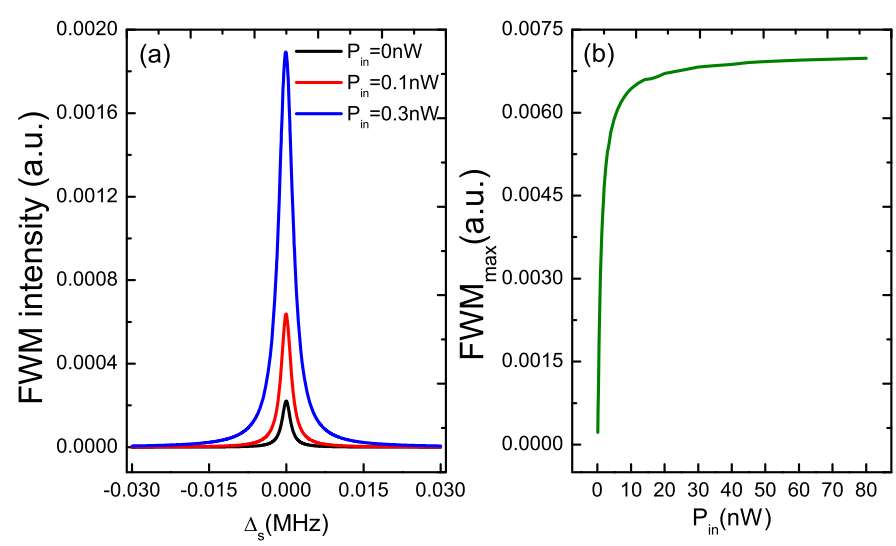

Figure 6 (a) The FWM intensity versus probe-cavity detuning for three pump field power $P_{\text {in }}=0,0.1,0.3 \mathrm{nW}$ under the red sideband. (b) FWM intensity as a function of pump power $P_{\text {in }}$

is driven simultaneously by a strong pump field with frequency $\omega_{p}$ and a weak probe field (with frequency $\omega_{s}$ ) on the red sideband, a new field (converted field) is generated by FWM at the frequency $2 \omega_{p}-\omega_{s}$. The conversion efficiency can be enhanced by increasing the power of the pump field. Experimental realization of this system [32-34] should make the application possible in the future.

\section{Conclusion}

We have demonstrated both electromagnetically induced transparency and microwave amplification in graphene optomechanical system under different detuning conditions. Destructive interference between the probe beam and the anti-Stokes field at red sideband leads to a transparency window in the probe transmission spectrum accompanying with a steep positive phase dispersion, giving rise to the corresponding slow light effect. Theoretical results show that an optically tunable delay of $0.4 \mathrm{~ms}$ of the transmitted probe beam. While at blue sideband, the constructive interference between the Stokes field and the probe field amplifies the weak probe field which suggests a optical transistor, and meanwhile a steep negative phase dispersion leads to fast-light effect. We further investigate the resonantly enhanced FWM in the coupled system, and the FWM intensity can be efficiently modulated by cavity-pump detuning and the pump power.

\section{Acknowledgements}

The authors gratefully acknowledge support from the National Natural Science Foundation of China (Nos: 11647001 and 11804004) and Anhui Provincial Natural Science Foundation (No: 1708085QA11).

\section{Funding}

Hua-Jun Chen is supported by the National Natural Science Foundation of China (Nos: 11647001 and 11804004) and Anhui Provincial Natural Science Foundation (No: 1708085QA11).

\section{Abbreviations}

FWM, Four-wave mixing; 2D, Two-dimensional; NMSs, Nanomechanical systems; C-OMS, Cavity-optomechanics systems; Q, Quality; OMS, Optomechanics systems; OMIT, Optomechanically induced transparency; OMIA, Optomechanically induced absorptionand; OMIR, optomechanically induced reflection.

Availability of data and materials

Not applicable. For all requests relating to the paper, please contact the author. 


\section{Authors' contributions}

HJC finished the main work of this paper, including conceiving of the idea, deducing the formulas, plotting the figures, and drafting the manuscript. All authors read and approved the final manuscript.

\section{Publisher's Note}

Springer Nature remains neutral with regard to jurisdictional claims in published maps and institutional affiliations.

Received: 31 January 2019 Accepted: 6 August 2019 Published online: 22 August 2019

\section{References}

1. Chen C, Rosenblatt S, Bolotin BKl, et al. Performance of monolayer graphene nanomechanical resonators with electrical readout. Nat Nanotechnol. 2009;4(12):861.

2. Eichler A, Moser J, Chaste J, et al. Nonlinear damping in mechanical resonators made from carbon nanotubes and graphene. Nat Nanotechnol. 2011;6(6):339.

3. Song $X$, Oksanen $M$, Sillanpää MA, et al. Stamp transferred suspended graphene mechanical resonators for radio frequency electrical readout. Nano Lett. 2011;12(1):198-202.

4. Chen C, Lee S, Deshpande VV, et al. Graphene mechanical oscillators with tunable frequency. Nat Nanotechnol. 2013;8(12):923.

5. Bunch JS, Van Der Zande AM, Verbridge SS, et al. Electromechanical resonators from graphene sheets. Science. 2007;315(5811):490-3.

6. Moser J, Güttinger J, Eichler A, et al. Ultrasensitive force detection with a nanotube mechanical resonator. Nat Nanotechnol. 2013;8(7):493.

7. Stapfner $\mathrm{S}$, Ost L, Hunger D, et al. Cavity-enhanced optical detection of carbon nanotube Brownian motion. Appl Phys Lett. 2013;102(15):151910.

8. Chiu HY, Hung P, Postma HWC, et al. Atomic-scale mass sensing using carbon nanotube resonators. Nano Lett. 2008:8(12):4342-6.

9. Chaste J, Eichler A, Moser J, et al. A nanomechanical mass sensor with yoctogram resolution. Nat Nanotechnol. 2012;7(5):301.

10. Singh V, Sengupta S, Solanki HS, et al. Probing thermal expansion of graphene and modal dispersion at low-temperature using graphene nanoelectromechanical systems resonators. Nanotechnology. 2010;21(16):165204.

11. Aspelmeyer M, Kippenberg TJ, Marquardt F. Cavity optomechanics. Rev Mod Phys. 2014;86(4):1391.

12. O'Connell AD, Hofheinz M, Ansmann M, et al. Quantum ground state and single-phonon control of a mechanical resonator. Nature. 2010;464(7289):697.

13. Teufel JD, Donner T, Li D, et al. Sideband cooling of micromechanical motion to the quantum ground state. Nature. 2011:475(7356):359.

14. Chan J, Alegre TPM, Safavi-Naeini AH, et al. Laser cooling of a nanomechanical oscillator into its quantum ground state. Nature. 2011:478(7367):89.

15. Barton RA, Storch IR, Adiga VP, et al. Photothermal self-oscillation and laser cooling of graphene optomechanical systems. Nano Lett. 2012;12(9):4681-6.

16. Peterson RW, Purdy TP, Kampel NS, et al. Laser cooling of a micromechanical membrane to the quantum backaction limit. Phys Rev Lett. 2016;116(6):063601.

17. Chen HJ. Manipulation of fast and slow light propagation by photonic-molecule optomechanics. J Appl Phys. 2018;124(15):153102

18. Rossi M, Mason D, Chen J, et al. Measurement-based quantum control of mechanical motion. Nature. 2018:563(7729):53.

19. Grudinin IS, Lee H, Painter O, et al. Phonon laser action in a tunable two-level system. Phys Rev Lett. 2010;104(8):083901.

20. Jing H, Özdemir SK, Lü XY, et al. PT-symmetric phonon laser. Phys Rev Lett. 2014;113(5):053604

21. Brooks DWC, Botter T, Schreppler S, et al. Non-classical light generated by quantum-noise-driven cavity optomechanics. Nature. 2012:488(7412):476.

22. Safavi-Naeini AH, Grölacher S, Hill JT, et al. Squeezed light from a silicon micromechanical resonator. Nature. 2013:500(7461):185

23. Purdy TP, Yu PL, Peterson RW, et al. Strong optomechanical squeezing of light. Phys Rev X. 2013;3(3):031012.

24. Agarwal GS, Huang S. Electromagnetically induced transparency in mechanical effects of light. Phys Rev A 2010;81(4):041803.

25. Weis S, Riviere R, Deleglise S, et al. Optomechanically induced transparency. Science. 2010;330(6010):1520-3.

26. Teufel JD, Li D, Allman MS, et al. Circuit cavity electromechanics in the strong-coupling regime. Nature. 2011;471(7337):204

27. Safavi-Naeini AH, Alegre TPM, Chan J, et al. Electromagnetically induced transparency and slow light with optomechanics. Nature. 2011;472(7341):69.

28. Karuza M, Biancofiore C, Bawaj M, et al. Optomechanically induced transparency in a membrane-in-the-middle setup at room temperature. Phys Rev A. 2013;88(1):013804

29. Zhou X, Hocke F, Schliesser A, et al. Slowing, advancing and switching of microwave signals using circuit nanoelectromechanics. Nat Phys. 2013;9(3):179.

30. Fan L, Fong KY, Poot $\mathrm{M}$, et al. Cascaded optical transparency in multimode-cavity optomechanical systems. Nat Commun. 2015;6:5850.

31. Massel F, Heikkilä TT, Pirkkalainen JM, et al. Microwave amplification with nanomechanical resonators. Nature. 2011;480(7377):351.

32. Weber P, Guttinger J, Tsioutsios I, et al. Coupling graphene mechanical resonators to superconducting microwave cavities. Nano Lett. 2014;14(5):2854-60. 
33. Singh V, Bosman SJ, Schneider BH, et al. Optomechanical coupling between a multilayer graphene mechanical resonator and a superconducting microwave cavity. Nat Nanotechnol. 2014;9(10):820.

34. Song X, Oksanen M, Li J, et al. Graphene optomechanics realized at microwave frequencies. Phys Rev Lett. 2014;113(2):027404

35. Huang S, Agarwal GS. Normal-mode splitting and antibunching in Stokes and anti-Stokes processes in cavity optomechanics: radiation-pressure-induced four-wave-mixing cavity optomechanics. Phys Rev A. 2010;81(3):033830.

36. Jia WZ, Wei LF, Li Y, et al. Phase-dependent optical response properties in an optomechanical system by coherently driving the mechanical resonator. Phys Rev A. 2015;91(4):043843.

37. Xu XW, Li Y. Controllable optical output fields from an optomechanical system with mechanical driving. Phys Rev A. 2015;92(2):023855

38. Jiang C, Cui Y, Liu H. Controllable four-wave mixing based on mechanical vibration in two-mode optomechanical systems. Europhys Lett. 2013;104(3):34004.

39. Regal CA, Teufel JD, Lehnert KW. Measuring nanomechanical motion with a microwave cavity interferometer. Nat Phys. 2008;4(7):555.

40. Boyd RW. Nonlinear Optics (Academic, 1992).

41. Gardiner CW, Zoller P. Quantum noise. 2nd ed. Berlin: Springer; 2000.

42. Woolley MJ, Doherty AC, Milburn GJ, et al. Nanomechanical squeezing with detection via a microwave cavity. Phys Rev A. 2008;78(6):062303.

Submit your manuscript to a SpringerOpen ${ }^{\circ}$ journal and benefit from:

- Convenient online submission

- Rigorous peer review

- Open access: articles freely available online

- High visibility within the field

- Retaining the copyright to your article

Submit your next manuscript at $\boldsymbol{\nabla}$ springeropen.com 